\title{
Oxidative Rearrangements of Tricyclic Vinylcyclobutane Derivatives
}

\author{
Juliane Grota, ${ }^{[a]}$ Jochen Mattay, ${ }^{*[a]}$ Krzysztof Piech, ${ }^{[b]}$ and Thomas Bally*[b]
}

\begin{abstract}
Three tricyclic vinylcyclobutanes (3-methylenetricyclo[5.3.0.0 $\left.0^{2,6}\right]$ decanes 1-3) have been subjected to ionization by photoinduced electron transfer in solution and by X-irradiation in Ar matrices. All three compounds undergo oxidative cycloreversion; the cleavage of the four-membered ring, however, occurs in a different direction depending on the presence of a methyl group in position 6 of the tricyclic framework. In those derivatives, cycloreversion is found to lead
\end{abstract}

to 1-methyl-8-methylene-1,6-cyclodecadiene radical cations $\left(5^{+}\right.$from $\mathbf{1}, \mathbf{8}^{+}$ from 2) which upon back electron transfer yield two different hydrocarbons ( 6 from $5^{\cdot+}, 9$ from $8^{+}$), depending on the configuration around the endocyclic double bonds of the respective

Keywords: cycloreversion • matrix isolation - photoinduced electron transfer - quantum chemical calculations $\cdot$ radical ions cyclodecadiene derivative. In the absence of a methyl group on C6, the cycloreversion leads to a radical cation complex between 1-methylenecyclopent-2-ene and cyclopentene $\left(\mathbf{1 2}^{+}\right)$ which appears to revert to $\mathbf{3}$ on back electron transfer. The intermediate radical cations $\mathbf{5}^{++}, \mathbf{8}^{\cdot+}$, and $\mathbf{1 2}^{+}$have been identified and characterized by $\mathrm{UV} / \mathrm{Vis}$ and IR spectra in Ar matrices. The mechanism of their formation is elucidated by quantum chemical calculations.

\section{Introduction}

Many familiar chemical reactions take a different, often unexpected course when the reactants are subjected to oneelectron oxidation or reduction. ${ }^{[1,2]}$ In other cases the expected products are formed, but the reactions are greatly accelerated (electron transfer catalysis ${ }^{[3]}$ ). In particular, many studies have been devoted to elucidate the kinetic and mechanistic effects of removing an electron from reagents involved in pericyclic reactions. ${ }^{[4]}$ Among those are the vinylcyclopropane $\mathrm{e}^{[5-8]}$ and vinylcyclobutane $\mathrm{e}^{[9,10]}$ rearrangements, the latter often in the context of a stepwise pathway for radical cation Diels-Alder reaction. ${ }^{[11-13]}$

When the vinylcyclobutane moiety is part of a rigid polycyclic framework, as it is the case in $\alpha$ - and $\beta$-pinene, then

[a] Dr. J. Grota, Prof. J. Mattay

Fakultät für Chemie, Universität Bielefeld

33501 Bielefeld (Germany)

E-mail: mattay@uni-bielefeld.de

[b] Dr. K. Piech, Prof. T. Bally

Departement de chimie, Université de Fribourg

1700 Fribourg (Switzerland)

E-mail: Thomas.Bally@unifr.ch

Supporting information for this article is available on the WWW under http://www.chemeurj.org/ or from the author. the sigmatropic rearrangement appears to be suppressed for steric reasons (in the case of $\beta$-pinene it would lead to a bridgehead olefin). Instead one-electron oxidation leads to products derived from ring-opened distonic radical cations. ${ }^{[14-17]}$
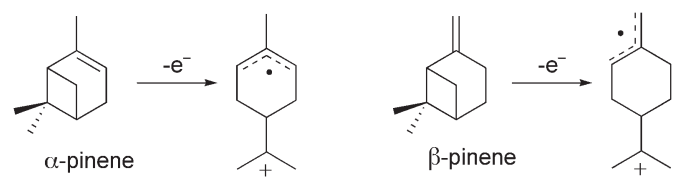

On the other hand, cyclobutanes have also been found to undergo cycloreversion on oxidation $;{ }^{[18-21]}$ the distonic radical cations which arise as primary products of the cleavage of a single bond in ionized cyclobutanes often show interesting ensuing rearrangements. ${ }^{[22,23]}$

Three tricyclic vinylcyclobutanes that we have recently synthesized have yielded some intriguing products upon photoinduced electron transfer. ${ }^{[2]}$ This paper is dedicated to the elucidation of these rearrangements which we have investigated by studying the reactive intermediates that are generated on ionization of the vinylcyclobutanes in Ar matrices, and by computational chemistry. In particular, we studied the three substrates $\mathbf{1}-\mathbf{3}$ shown below. 


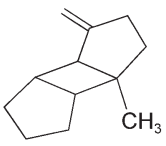

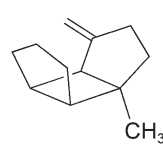

2

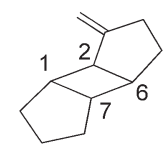

3

\section{Methods}

Syntheses: The tricyclic vinylcyclobutanes used in this study were synthesized according to previously published procedures. ${ }^{[25]}$

Matrix isolation: The substrates were premixed in a 1:1000 ratio with a gas mixture consisting of $85 \% \mathrm{Ar}, 15 \% \mathrm{~N}_{2}$ (which was added to improve the optical quality of the matrices) and $0.1 \% \mathrm{CH}_{2} \mathrm{Cl}_{2}$ (which acts as an electron scavenger). This mixture was deposited slowly onto a CsI window held at $20 \mathrm{~K}$ in a closed-cycle cryostat. After that the sample was cooled to the lowest temperature attainable by the cryostat $(6$ or $10 \mathrm{~K}$, depending on the adsorbant used in the second expansion stage), and exposed to X-irradiation (W anode, $40 \mathrm{kV}, 20 \mathrm{~mA}$ ) for different intervals of time.

Spectroscopy: Electronic absorption (EA) spectra were recorded between 200 and $2000 \mathrm{~nm}$ on a Perkin-Elmer Lambda 900 instrument with $1 \mathrm{~nm}$ resolution. For IR spectra we employed a Bomem DA3 FT-interferometer which was operated at a resolution of $0.5 \mathrm{~cm}^{-1}$ with an MCT detector. Usually 256 scans were accumulated for each IR spectrum.

Computations: Except where noted otherwise we employed the B3LYP density functional method (Becke's three-parameter hybrid exchange functional ${ }^{[26]}$ combined with the Lee-Yang-Parr correlation functional ${ }^{[27]}$ ) with the $6-31 \mathrm{G}^{*}$ basis set as implemented in the Gaussian 98 program package ${ }^{[28]}$ for geometry optimizations and frequency calculations. Excited state calculations were carried out by time-dependent response theory, based again on density functional methods (the so-called TDDFT method), ${ }^{[29]}$ as implemented in G03. ${ }^{[30]}$ Thereby the B3LYP functional and the $6-31 \mathrm{G}^{*}$ basis set were again used.

\section{Experimental Results}

Figures 1-3 document the spectral changes observed on Xirradiation (black curves) and on subsequent selective photolyses (red and blue curves) in the UV/Vis/NIR and in the IR spectroscopical range. These spectra will be discussed separately for each compound in the following three subsections.

Compound 1: In the case of 1, ionization leads to a broad band peaking at $675 \mathrm{~nm}$, in addition to a range of sharper bands with $\lambda_{\max }$ of 460,400 und $325 \mathrm{~nm}$. Photolysis at the low energy edge of the visible band $(>780 \mathrm{~nm})$ leads to a decrease at $>700 \mathrm{~nm}$ and a concomitant increase at the high-energy edge of this band (red curve in Figure 1). Similar behavior is observed for the other bands in the spectrum. Conversely, irradiation through a $578 \mathrm{~nm}$ interference filter bleaches mainly the high-energy part of the broad visible band while the NIR absorptions increase (blue curve in Figure 1).

Similarly, the IR difference spectra show new peaks upon $\mathrm{X}$-irradiation (black curve) while subsequent photolysis at $>780 \mathrm{~nm}$ leads to changes that involve mainly very closelying peaks (see, e.g., the bands at 1350 and $1380 \mathrm{~cm}^{-1}$ in
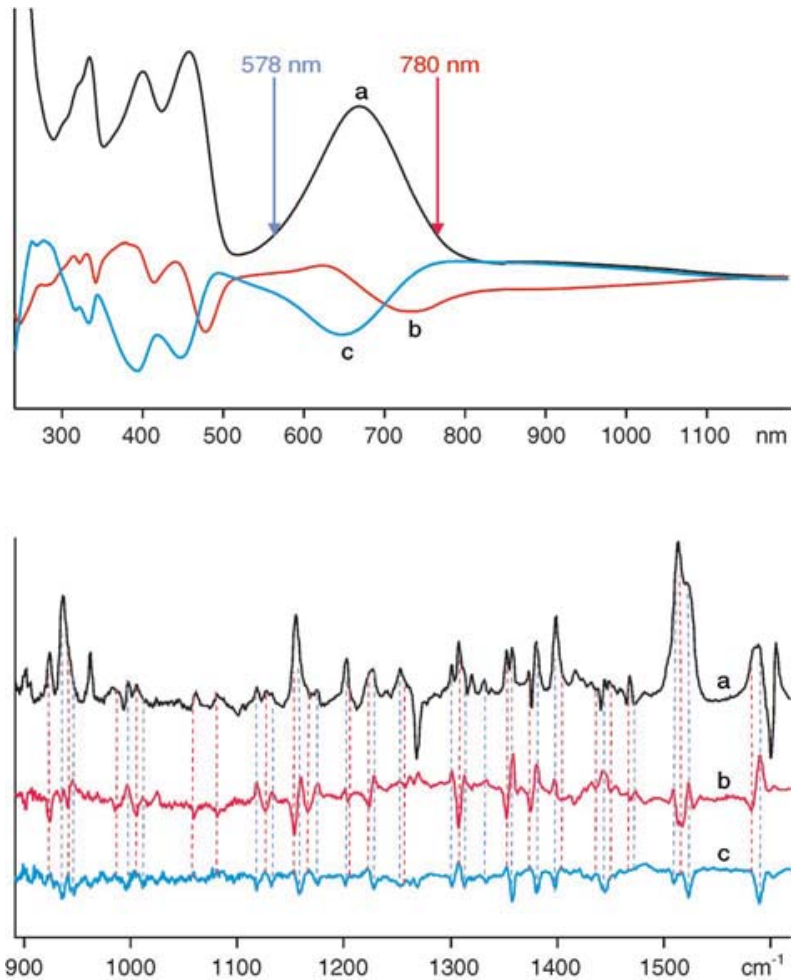

Figure 1. Electronic absorption (top) and IR difference spectra (bottom) for the X-irradiation of $\mathbf{1}$ in Ar matrices (black traces, a) and for subsequent photolyses at $>780 \mathrm{~nm}$ (red traces, b) and then at $578 \mathrm{~nm}$ (blue traces, c).

the red trace). The IR difference spectrum for $578 \mathrm{~nm}$ irradiation (blue trace) is almost a mirror image of the red trace, although the increasing peaks are quite weak in this case, indicating only partial reversibility of the process.

From these findings, we conclude that ionization of $\mathbf{1}$ leads to two species that can be partially interconverted. The great similarity of these species, as it becomes evident from the difference spectra, indicates that they have a similar structure. Indeed, the calculations presented in the following sections show that the spectra can be well explained in terms of two conformers of the 1-methyl-8-methylene-1,6cyclodecadiene radical cation that arise by cycloreversion of the four-membered ring in ionized $\mathbf{1}$.

Compound 2: For the syn-tricyclodecane derivative 2, the picture is quite similar in that we see again a new broad band peaking at the long wavelength edge of the visible range, accompanied by some sharper peaks between 300 and $500 \mathrm{~nm}$ (black spectrum in Figure 2). In addition there appears to be a weak, broad absorption that tails into the near IR range. Attempts to selectively bleach that absorption by irradiation at $>970 \mathrm{~nm}$ led to a difference spectrum (red trace in Figure 2) that shows a weak decrease in the NIR accompanied by a further growth of the band peaking at $750 \mathrm{~m}$. Bleaching at the low-energy edge of this band led to its complete disappearance (blue trace) whereby no new absorptions arise above $400 \mathrm{~nm}$. 

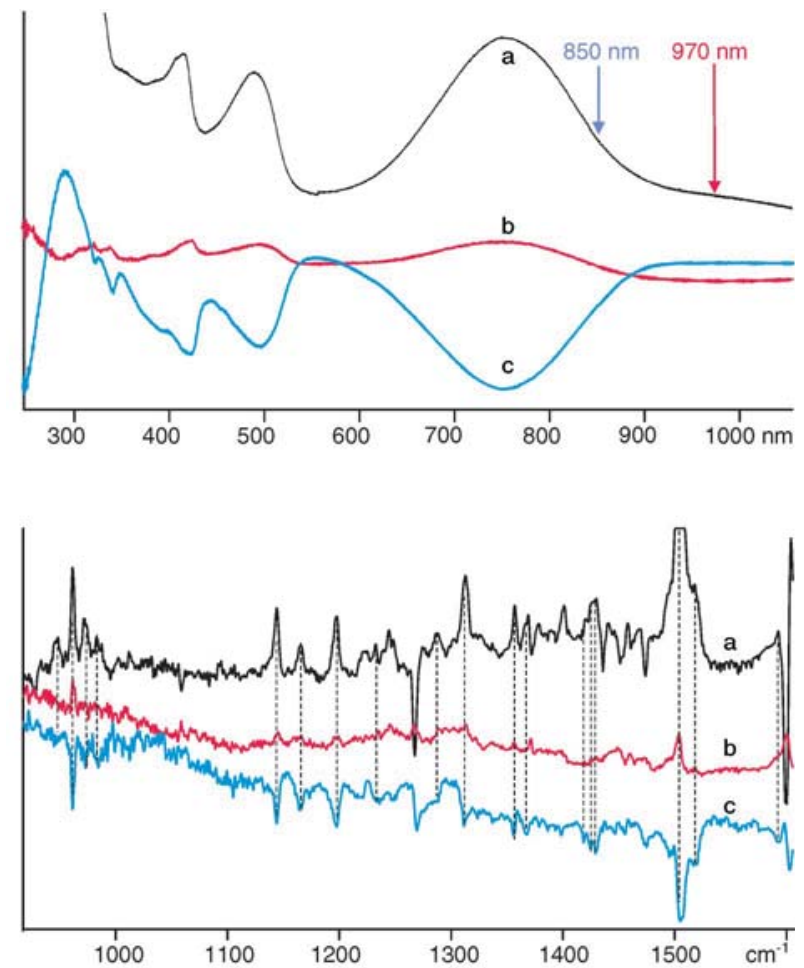

Figure 2. Electronic absorption (top) and IR difference spectra (bottom) for the X-irradiation of $\mathbf{2}$ in Ar matrices (black traces, a) and for subsequent photolyses at $>970 \mathrm{~nm}$ (red traces, b) and then at $>850 \mathrm{~nm}$ (blue traces, c).

The IR difference spectra give a congruent picture. Unfortunately, the one for $>970 \mathrm{~nm}$ bleaching (red trace) is too weak to furnish useful information about the identity of the product that absorbs in the NIR, it only confirms that a small additional amount of the main product of ionization of 2 is formed in the process. Finally, bleaching at $>850 \mathrm{~nm}$ (blue trace) leads to a pronounced decrease of the peaks of the main ionization product. Surprisingly, no IR peaks were found that increase during this irradiation, so this experiment does not reveal much information about the fate of the primary product of ionization on photolysis. The calculations discussed below will show that this product is again a 1-methyl-8-methylene-1,6-cyclodecadiene radical cation, that is, isomer $\mathbf{2}$ also undergoes spontaneous cycloreversion on ionization in Ar.

Compound 3: Finally, the unmethylated compound $\mathbf{3}$ was subjected to the same experimental protocol. Thereby distinctly different spectra were obtained compared with the previous two cases, indicating that a different type of product is formed in this case. The main product of ionization distinguishes itself by a double-humped band that spans most of the visible range and extends up to $1100 \mathrm{~nm}$ into the NIR (black spectrum in Figure 3). NIR irradiations were quite inefficient and did not reveal any composite bands. On photolysis at $>830 \mathrm{~nm}$, the band peaking at this wavelength
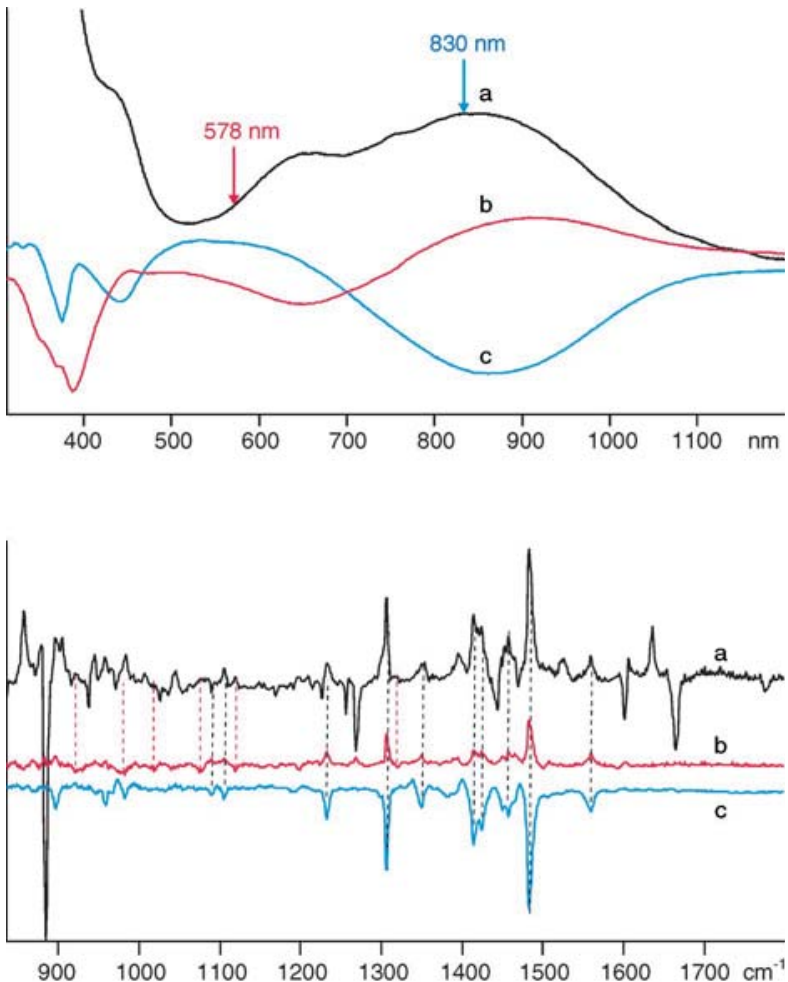

Figure 3. Electronic absorption (top) and IR difference spectra (bottom) for the X-irradiation of $\mathbf{3}$ in Ar matrices (black traces, a) and for subsequent photolyses at $578 \mathrm{~nm}$ (red traces, b) and then at $830 \mathrm{~nm}$ (blue traces, c).

was bleached, together with some sharper peaks at 450 and $380 \mathrm{~nm}$, while a broad absorption at 500-600 nm increased (blue trace). The shoulder at $650 \mathrm{~nm}$ did not seem to be affected by this irradiation, so we exposed the sample to photolysis at $578 \mathrm{~nm}$, the result of which is shown in the red trace. Indeed, this irradiation leads to the decrease of a broad band peaking at about $650 \mathrm{~nm}$ and the concomitant increase of the $830 \mathrm{~nm}$ band (the maximum of which is shifted to longer wavelength in the difference spectrum).

The corresponding IR spectra show the formation (black trace) and bleaching of a species with a distinct IR spectrum (blue trace), but the difference spectrum for $578 \mathrm{~nm}$ irradiation does not reveal much information on the species that is bleached in this process, it only shows that more of the main product of ionization of $\mathbf{3}$ is formed. The calculations presented below will demonstrate that this main product is a radical cation complex between 1-methylenecyclopent-2-ene and cyclopentene, so $\mathbf{3}$ also undergoes cycloreversion, albeit in a different way in that the two bonds which remain intact on ionization of $\mathbf{1}$ and $\mathbf{2}$ are separated in $\mathbf{3}$.

In the following section we will offer assignments of the spectra mentioned above and try to explain the chemistry that is expressed in these spectra in terms of the potential energy surfaces of the radical cations that are formed as primary species on $\mathrm{X}$-irradiation of compounds $\mathbf{1 - 3}$ in Ar. 


\section{Calculations and Discussion}

Compound 1: In spite of its rigidity, the tricyclic carbon frame of hydrocarbon $\mathbf{1}$ has some conformational freedom, especially with regard to the peripheral methylene groups which can assume an endo- or an exo-position with regard to the rest of the molecule. This gives rise to four conformers depicted in Figure 4, the relative free energies of which are listed in Table 1

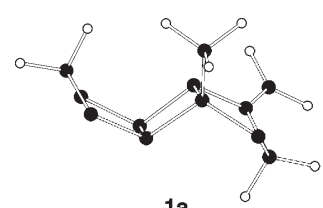

$1 a$

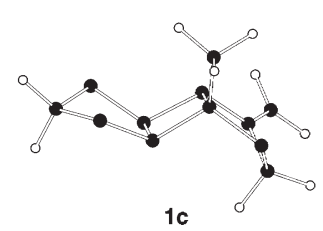

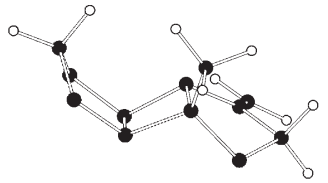

$1 \mathrm{~b}$

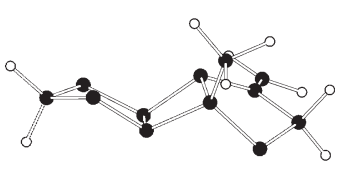

$1 d$
Figure 4. Four conformers of $\mathbf{1}$ (from B3LYP/6-31G* calculations. Note that many hydrogen atoms in the ball-and-stick models are left away for clarity).

Table 1. Relative free energies (from B3LYP/6-31G* calculations) and equilibrium composition of $\mathbf{1} \mathbf{a}-\mathbf{d}$.

\begin{tabular}{rcc}
\hline Conformer & $\Delta G\left[\mathrm{kcal} \mathrm{mol}^{-1}\right]$ & \% in equilibrium at $298 \mathrm{~K}$ \\
\hline $\mathbf{1 a}$ & $(0)$ & 81.2 \\
$\mathbf{1 b}$ & 1.07 & 13.3 \\
$\mathbf{1 c}$ & 1.64 & 5.1 \\
$\mathbf{1 d}$ & 3.09 & 0.4 \\
\hline
\end{tabular}

These predictions are in good agreement with the NMR spectra of $\mathbf{1}^{[24,31]}$ so we assume that $\mathbf{1}$ is present predominantly as conformers $\mathbf{1 a}$ and $\mathbf{1 b}$.

Upon ionization of $\mathbf{1}$ the five-membered ring carrying the exo-methylene group becomes planar. Hence $\mathbf{1 a}$ and $\mathbf{1 b}$ relax to the same radical cation, $\mathbf{1} \mathbf{a}^{\cdot+}$, whereas ionization of $\mathbf{1 c}$ and $\mathbf{1 d}$ leads to different conformers of $\mathbf{1}^{\cdot+}$. As these are both formed from minor constituents of $\mathbf{1}$ and lie about $3.5 \mathrm{kcal} \mathrm{mol}^{-1}$ above $\mathbf{1 a}^{++}$on a $298 \mathrm{~K}$ enthalpy scale, we will not consider them further. Figure 5 shows that the HOMO of $1 \mathbf{a}$ extends significantly into the four-membered ring bond which is antiperiplanar to the olefinic $\pi-\mathrm{MO}(\mathrm{C} 2-\mathrm{C} 6)$. Therefore it comes as no surprise that ionization does not only lead to elongation of the double bond, but also to that of the antiperiplanar $\sigma$ bond, while the other bonds in the four-membered ring retain approximately the lengths they had in neutral 1a. This leads to a trapezoidal structure which is typical of 1,2-disubstituted cyclobutane radical cations. $^{[32]}$

Thus one would assume that the $\mathrm{C} 1-\mathrm{C} 2$ bond is poised to break in $\mathbf{1} \mathbf{a}^{\cdot+}$, but a potential energy scan wherein this bond
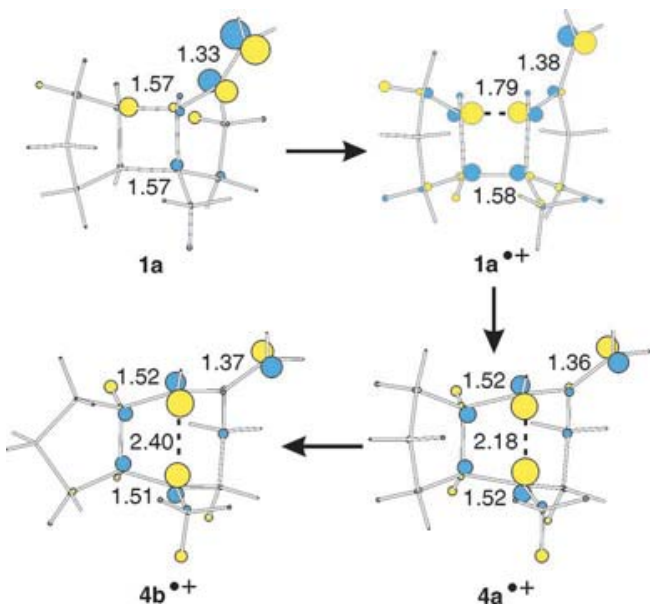

Figure 5. HOMOs of $\mathbf{1 a}, \mathbf{1} \mathbf{a}^{++}, \mathbf{4 a}^{\cdot+}$ and $\mathbf{4} \mathbf{b}^{\cdot+}$. Numbers indicate bond lengths in $\AA$.

was further stretched led steadily uphill without yielding a stable product (the distonic radical cation that would be attained in this process collapses back to $\mathbf{1 a}^{\cdot+}$ on optimization). In contrast, we found that cleavage of the adjacent C2-C6 bond proceeds with almost no activation, via a transition state with a kite-shaped cyclobutane moiety, ${ }^{[32]}$ to yield the radical cation $\mathbf{4} \mathbf{a}^{+}$which again has a trapezoidal cyclobutane frame, but this time perpendicular to that of $\mathbf{1 a}^{\cdot+}$. The spin distribution in the singly occupied HOMO of $\mathbf{4} \mathbf{a}^{+}$(cf. Figure 5) indicates that this species may also be regarded as an intramolecular complex between an allyl radical and a trialkylcarbonium cation.

In an attempt to induce net cycloreversion of $\mathbf{1}^{\mathbf{a}^{+}}$we subjected the $\mathrm{C} 1-\mathrm{C} 7$ bond in $\mathbf{4 a}^{\cdot+}$ to stepwise dilation. However, this resulted once again in an uphill process the result of which was that the $\mathrm{C} 2-\mathrm{C} 6$ bond was re-formed, which means that cleavage of the $\mathrm{C} 1-\mathrm{C} 7$ bond led to a radical cation that does not profit from stabilization by allylic resonance (as $\mathbf{1} \mathbf{a}^{\cdot+}$ and $\mathbf{4} \mathbf{a}^{++}$, cf. Figure 5) and collapses back to $\mathbf{4} \mathbf{a}^{\cdot+}$ upon optimization.

On the other hand we found that $\mathbf{4} \mathbf{a}^{\cdot+}$ may be converted in a low-barrier process into another conformer, $\mathbf{4} \mathbf{b}^{\cdot+}$ which differs in the conformation of the saturated five-membered ring and has a somewhat longer $\mathrm{C} 2-\mathrm{C} 6$ bond (cf. Figure 5, the same species is also formed directly by decay of the two minor conformers of $\mathbf{1}^{+}$). In contrast to $\mathbf{4} \mathbf{a}^{\cdot+}$, stretching of the $\mathrm{C} 1-\mathrm{C} 7$ bond in $\mathbf{4} \mathbf{b}^{+}$led to nearly activationless (and quite exothermic) cleavage to yield the radical cation of 1 methyl-8-methylene-(E,E)-1,6-cyclodecadiene $\left(\mathbf{5} \mathbf{b}^{\cdot+}\right)$. When we explored the conformational space of $\mathbf{5}^{+}$we found another minimum, $\mathbf{5} \mathbf{c}^{\cdot+}$, which differs from $\mathbf{5} \mathbf{b}^{\cdot+}$ mainly in the conformation of the trimethylene bridge. However, as seen in Figure 6, this change affects also the distance between the diene and the olefin moieties which are bound in an intermolecular $\pi$-complex cation. ${ }^{[19]}$ This binding is less strong in $\mathbf{5} \mathbf{c}^{\mathbf{}+}$ which is therefore about $3 \mathrm{kcal} \mathrm{mol}^{-1}$ less stable than $\mathbf{5}^{\cdot+}$. Also, the $\mathbf{5 b}^{\cdot+} \rightarrow \mathbf{5} \mathbf{c}^{\cdot+}$ rearrangement involves a predicted barrier of $8.8 \mathrm{kcal} \mathrm{mol}^{-1}$, that is, it would not proceed 


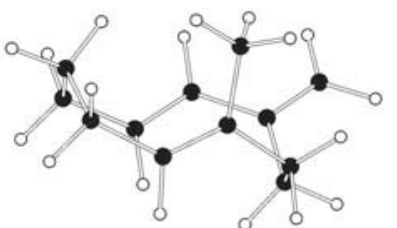

$5 b^{*+}$

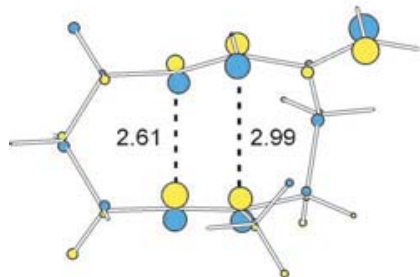

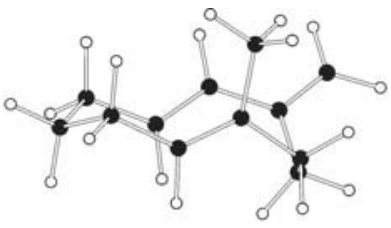

$5 c^{\bullet+}$

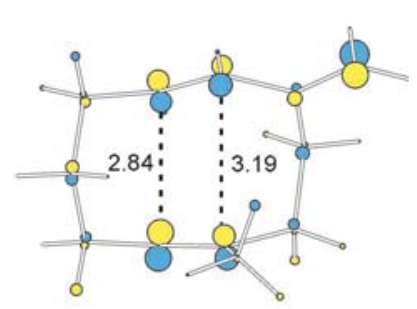

Figure 6. Structures and singly occupied MOs of 1-methyl-8-methylene1,6-cyclodecadiene radical cations $\mathbf{5 b}^{\cdot+}$ and $\mathbf{5} \mathbf{c}^{\cdot+}$. Numbers indicate distances in $\AA$.

spontaneously from thermalized $\mathbf{5} \mathbf{b}^{+}$at cryogenic temperatures.

Figure 7 sums up the sequence of events discussed above in the form of an enthalpy diagram. From this we conclude that the first species to be detectable after ionization of $\mathbf{1}$ is likely to be radical cation $\mathbf{5} \mathbf{b}^{\cdot+}$ because the small activation barriers that exist on the way to this species are easily overcome, either with the thermal energy at room temperature, or with the excess energy that is imparted onto the incipient radical cations formed by charge transfer from ionized Ar in the cryogenic experiments. It is not impossible that enough excess energy is available to induce also the conformational rearrangement of $\mathbf{5} \mathbf{b}^{\cdot+}$ to $\mathbf{5} \mathbf{c}^{\cdot+}$, and the experiments described in the Experimental Results section seem to indicate that this is indeed the case, because ionization of $\mathbf{1}$ results in formation of two species that have very similar electronic and vibrational absorption spectra, and these spectra are in good accord with those that are predicted for $\mathbf{5} \mathbf{b}^{\cdot+}$ and $\mathbf{5} \mathbf{c}^{\cdot+}$, as shown in Figure 8 (IR) and Table 2 (UV/Vis/NIR).

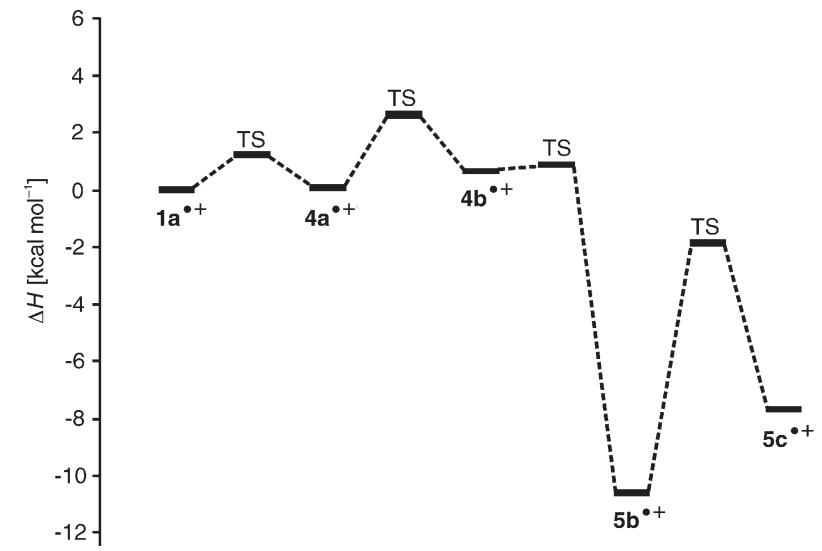

Figure 7. Enthalpy diagram for the decay of $\mathbf{1} \mathbf{a}^{\cdot+}$.
It can be assumed that the small barriers separating ionized $\mathbf{1}$ from $\mathbf{5}^{+}$are also readily overcome in PET experiments involving $\mathbf{1}$. Therefore the question arises in what way the main product which was found in these experiments, ${ }^{[24]}$ that is, 1-methyl-8-methylenetricyclo[4.4.0.0. $\left.0^{2,7}\right]$ decane $(6)$, is formed.

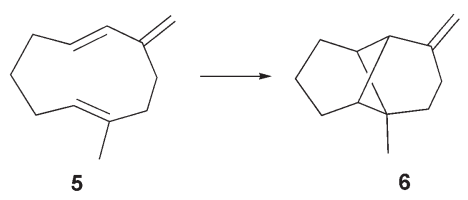

In principle 6 could arise by cross-cycloaddition of $\mathbf{5}^{+}$to $\mathbf{6}^{+}$, but calculations indicate that this reaction is thermodynamically unfeasible because $6^{+}$lies $12.7 \mathrm{kcal} \mathrm{mol}^{-1}$ above $\mathbf{5} \mathbf{b}^{\cdot+}$ on a free energy scale. ${ }^{[33]}$ Conversely, the reduction of $\mathbf{5} \mathbf{b}^{+}$to the triplet state of $\mathbf{5}$ is strongly exergonic $(\Delta G=$ $-125.7 \mathrm{kcal} \mathrm{mol}^{-1}$ according to B3LYP/6-31G*). As the triplet sensitized [2+2] cycloaddition of a 8-methylene-1,6-cyclodecadiene derivative to give the corresponding 8-methylenetricyclo[4.4.0.0 $\left.0^{2,7}\right]$ decane is known, ${ }^{[34]}$ we assume that $\mathbf{6}$ is formed after back electron transfer from $\mathbf{5}^{\cdot+}$ to the sensitizer radical anion.

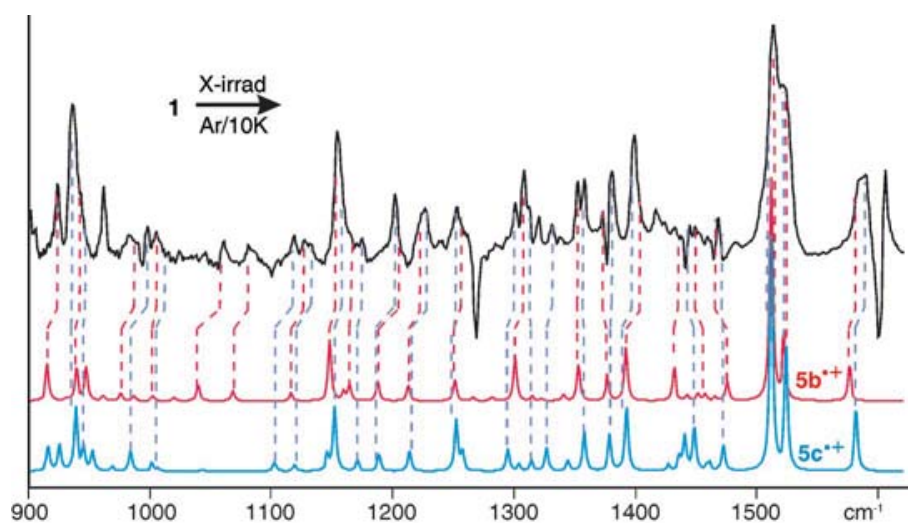

Figure 8. IR difference spectrum from Figure 1 (top trace), compared with spectra calculated by B3LYP/6-31G* for $\mathbf{5 b}^{++}$and $\mathbf{5} \mathbf{c}^{*+}$ (bottom two traces).

Table 2. Optical transitions and transition moments for $\mathbf{5} \mathbf{b}^{-+}$and $\mathbf{5} \mathbf{c}^{\cdot+}$ according to TD-B3LYP calculations.

\begin{tabular}{lcc}
\hline Compound & Wavelength & Transition moment \\
\hline $\mathbf{5 b}^{++}$ & 323 & 0.0148 \\
& 341 & 0.0053 \\
& 372 & 0.0877 \\
& 418 & 0.0563 \\
& 598 & 0.0843 \\
$\mathbf{5 c}^{+}$ & 356 & 0.0028 \\
& 393 & 0.0095 \\
& 416 & 0.0101 \\
& 488 & 0.0266 \\
& 779 & 0.0887 \\
\hline
\end{tabular}


Compound 2: The syn-isomer of $\mathbf{1}$, compound 2, exists also in different conformations, but, to simplify the calculations, only the lowest-energy conformer $\mathbf{2}$ a was considered (where one of the peripheral methylene groups is in an endo- and the other in an exo-position). Upon ionization, this species also relaxes to a structure $\mathbf{2}^{\cdot+}$ with a trapezoidal cyclobutane moiety, but, as in the case of $\mathbf{1}$, the bond which is most easily broken is not the one that is lengthened on ionization. Instead, extension of the $\mathrm{C} 2-\mathrm{C} 6$ bond leads to radical cation $\mathbf{7} \mathbf{a}^{+}$, the analogue of $\mathbf{4 a}^{\cdot+}$ above (see Figure 9). Again, this latter compound undergoes a facile conformational change to a structure $\mathbf{7} \mathbf{b}^{\cdot+}$ in which the methylene group in the intact five-membered ring adopts an exo-position. In this case, this small conformational change entrails, however, a complete cleavage of the $\mathrm{C} 2-\mathrm{C} 6$ bond which results in a truly distonic radical cation with an allylic moiety that carries most of the spin, while the LUMO (which can be regarded as the "charge-bearing" MO) is localized mostly on

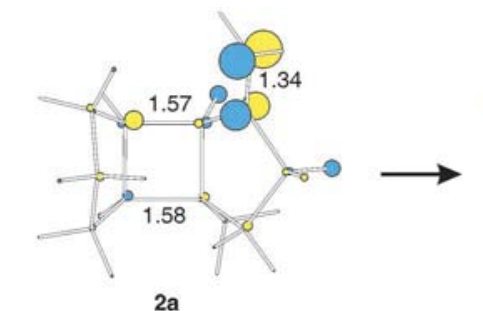

$2 a$
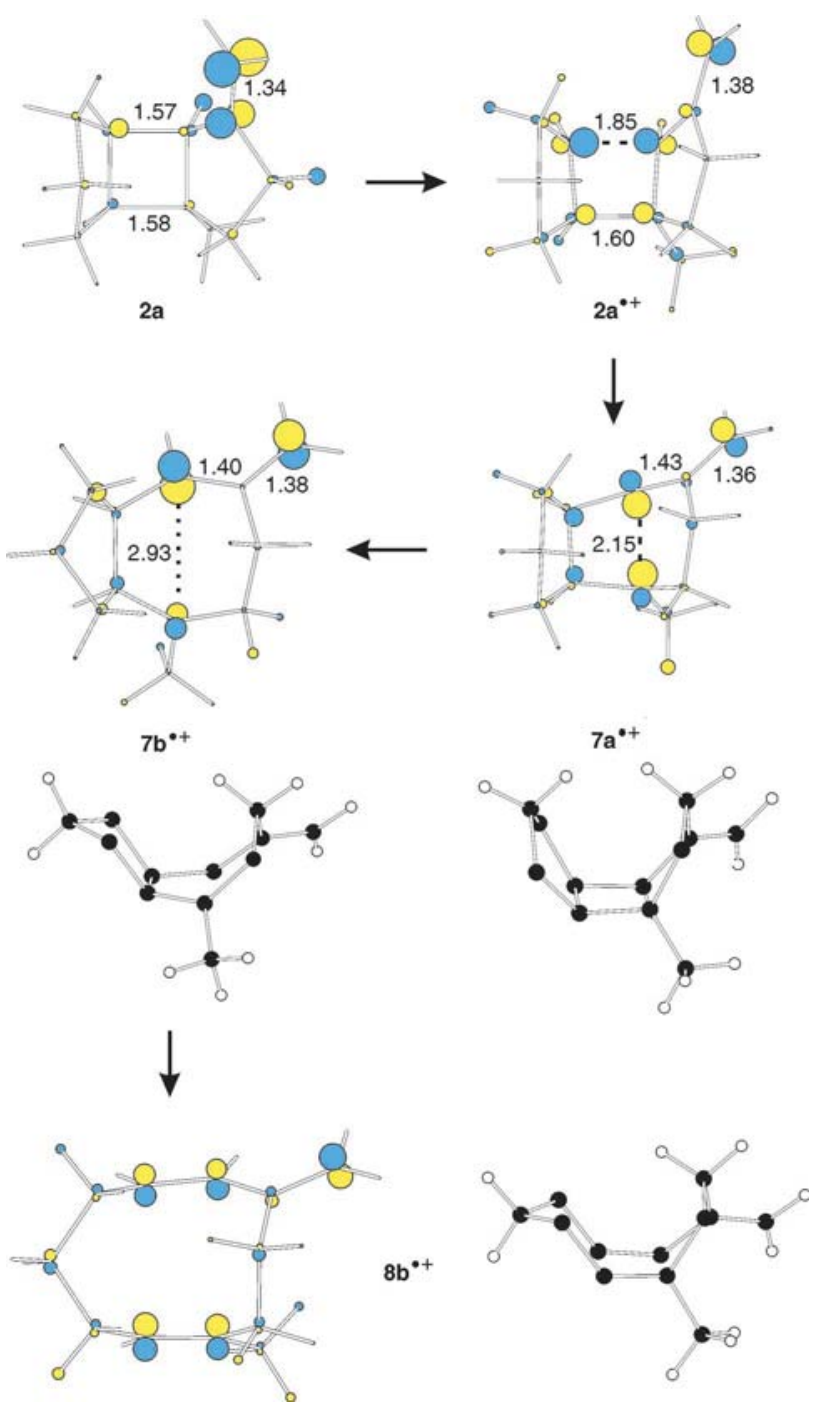

Figure 9. Species on the decay path of $\mathbf{2 a}$ on ionization. Numbers indicate bond lengths in $\AA$. The MO shown is the HOMO, which is singly occupied in the radical cations. Some hydrogen atoms are omitted in the ball-and-stick structures for clarity.
C6. Also $\mathbf{7} \mathbf{b}^{++}$is about $2 \mathrm{kcalmol}^{-1}$ more stable than $\mathbf{7} \mathbf{a}^{\mathbf{}^{+}}$, so that any further chemistry must be assumed to originate from $\mathbf{7} \mathbf{b}^{++}$(Figure 9).

Indeed, cleavage of the $\mathrm{C} 1-\mathrm{C} 7$ bond occurs, as in $\mathbf{4} \mathbf{b}^{\mathbf{}}$, with no activation on an enthalpy scale (see Figure 10) and leads to the much more stable radical cation of 1-methyl-8methylene- $(Z, Z)-1,6$-cyclodecadiene $\left(\mathbf{8} \mathbf{b}^{\cdot+}\right)$ of which we were only able to find a single stable conformer. Thus, as in the case of the anti isomer $\mathbf{1}$, ionization of $\mathbf{2}$ leads to complete cycloreversion (see Figure 10), and the IR and UV spectra obtained after X-irradiation of Ar matrices containing $\mathbf{1}$ are indeed in good agreement with those predicted for $\mathbf{8 b}^{++}$, as shown in Figure 11.

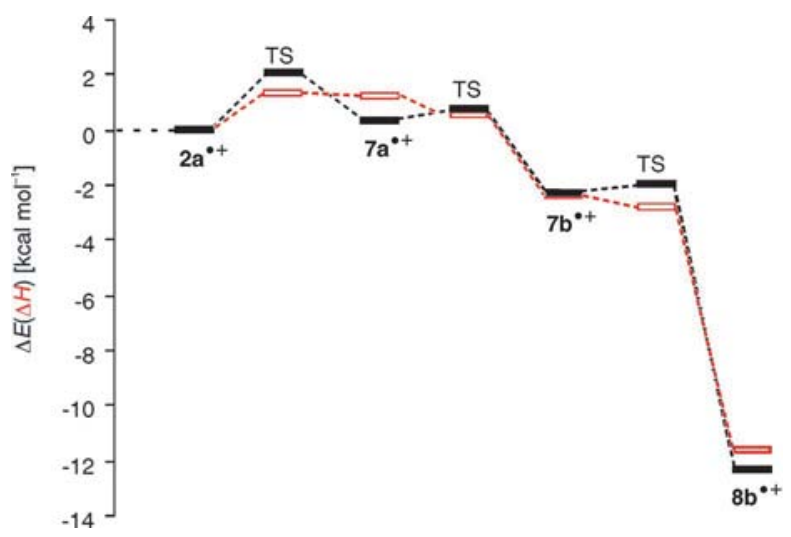

Figure 10. Energy (black, solid bars) and enthalpy diagram (red) for the decay of $\mathbf{2} \mathbf{a}$ on ionization.

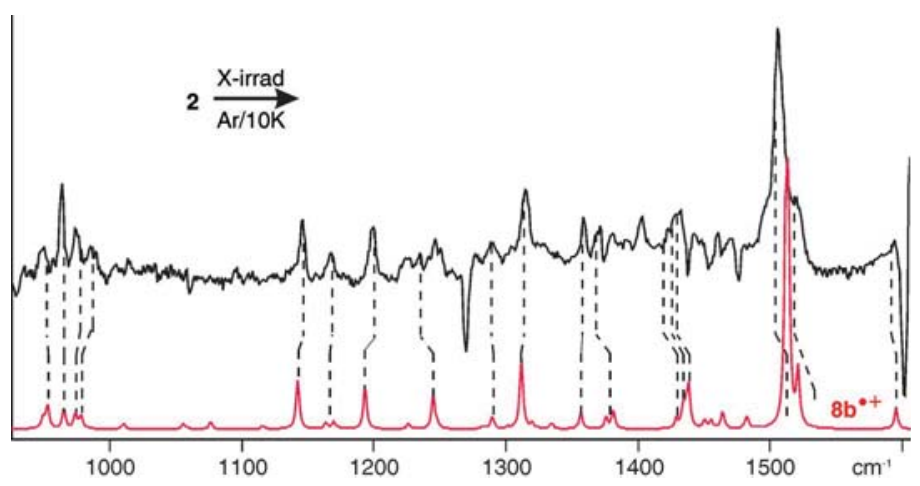

Figure 11. IR difference spectrum from Figure 2 (top) compared with that calculated by B3LYP/6-31G* for $\mathbf{8 b}^{*+}$ (bottom).

In the PET experiments with 2 the products that were found are 1, 6, and, in addition, bicyclic hydrocarbon 9. ${ }^{[24]}$ We had previously argued that cross-cycloaddition of decadiene $\mathbf{5} \mathbf{b}^{+}$occurs after reduction to the triplet state, and we presume that the same is happening in the case of $\mathbf{8} \mathbf{b}^{\cdot+}$.

Suprafacial cyclization of $\mathbf{8 b}^{\cdot+}$ to $\mathbf{1}^{++}$(or $\mathbf{4}^{+}$) is also

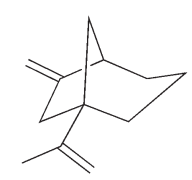

9 
strongly endothermic and requires cis-trans isomerizations of both endocyclic double bonds. Such isomerizations are known to take place on triplet sensitization of 8-methylene$(Z, Z)-1,6$-cyclodecadienes, ${ }^{[3]}$ hence we believe that this process occurs also on the triplet surface, in competition with cross-cycloaddition to yield 6. Most probably, 2 is also regenerated in this process, but this cannot be proven experimentally.

In contrast, there is no precedent for the formation of 9 in triplet-sensitized processes which suggests that this compound is at least pre-formed before back electron transfer occurs. Although the formation of $\mathbf{9}^{++}$from $\mathbf{2} \mathbf{a}^{+}$is thermochemically feasible according to our calculations, it is endergonic by over $7 \mathrm{kcalmol}^{-1}$ from $\mathbf{8 \mathbf { b } ^ { + + }}$ to which $\mathbf{2}^{\cdot+}$ decays spontaneously. Thus, the first reaction step leading from $\mathbf{2} \mathbf{a}^{\mathbf{a}^{+}}$to $\mathbf{9}^{\cdot+}$ would have to compete with cleavage to $\mathbf{7} \mathbf{a}^{\cdot+}$ which has a negligible activation enthalpy (the ensuing decay of $\mathbf{7} \mathbf{a}^{++}$to $\mathbf{9}^{\cdot+}$ is also practically barrierless). In computational experiments, we explored all sorts of feasible rearrangements of $\mathbf{2}^{\cdot+}$ that may ultimately lead to $\mathbf{9}^{\cdot{ }^{+[35]}}$ but none of them comes even close to compete with its decay to $\mathbf{7} \mathbf{a}^{\cdot+}$.

Compound 3: In PET experiments 3, which differs from 1 only by the lack of a methyl group on C6, showed only a very slow decomposition which was not accompanied by the formation of products that were detectable by GC. This could be due to the fact that the barriers for "productive" rearrangements are too high for these processes to compete with back electron transfer. Alternatively the volatility of the products is such that they either do not make it through the GC column (i.e., polymers) or that they appear together with the solvent. In contrast, ionization of $\mathbf{3}$ in an Ar matrix led to at least three products the major one of which could be clearly identified by its IR spectrum.

Compound $\mathbf{3}$ exists also in four conformations $\mathbf{3 a} \mathbf{a}-\mathbf{d}$ which closely correspond to those of $\mathbf{1}$ (cf. Figure 4). Conformer 3a is largely predominant ( $94 \%$ in the mixture at $298 \mathrm{~K}$ ) while $\mathbf{3 b}$ almost makes up for the rest. These two conformers give the same radical cation $\mathbf{3} \mathbf{a}^{+}$on ionization, but attempts to stretch the $\mathrm{C} 1-\mathrm{C} 2$ or the $\mathrm{C} 2-\mathrm{C} 6$ bonds in $\mathbf{3} \mathbf{a}^{+}$ did not lead to stable products. However, flipping over the methylene group in the saturated five-membered ring leads to a conformer $\mathbf{3 b}^{+}$(Figure 12) which is $2.9 \mathrm{kcal} \mathrm{mol}^{-1}$ higher in free energy but which is more amenable to cleavage of the bonds that are in $\alpha, \beta$-position to the exocyclic methylene group.

Cleavage of the $\mathrm{C} 2-\mathrm{C} 6$ bond, analogous to $4 \mathbf{a}^{\cdot+}$ lead to a species $\mathbf{1 0}^{-+}$that lies $6.7 \mathrm{kcalmol}^{-1}$ higher in free energy than $\mathbf{3} \mathbf{b}^{++}$and is not protected from collapse back to $\mathbf{3} \mathbf{b}^{+}$. Stretching the $\mathrm{C} 1-\mathrm{C} 7$ bond in $\mathbf{1 0}^{-+}$led to re-formation of the $\mathrm{C} 2-\mathrm{C} 6$ bond, as it had in $\mathbf{4} \mathbf{a}^{\cdot+}$, and did not lead to a stable product. Conversely, $\mathrm{C} 1-\mathrm{C} 2$ dissociation in $\mathbf{3 b}^{++}$ which involves an activation enthalpy of only $6 \mathrm{kcal} \mathrm{mol}^{-1}$ leads to the distonic radical cation $\mathbf{1 1}^{-+}$which in turn undergoes spontaneous cleavage of the $\mathrm{C} 6-\mathrm{C} 7$ bond to yield the radical cation complex $\mathbf{1 2}^{+}$between 3 -methylenecyclopen-
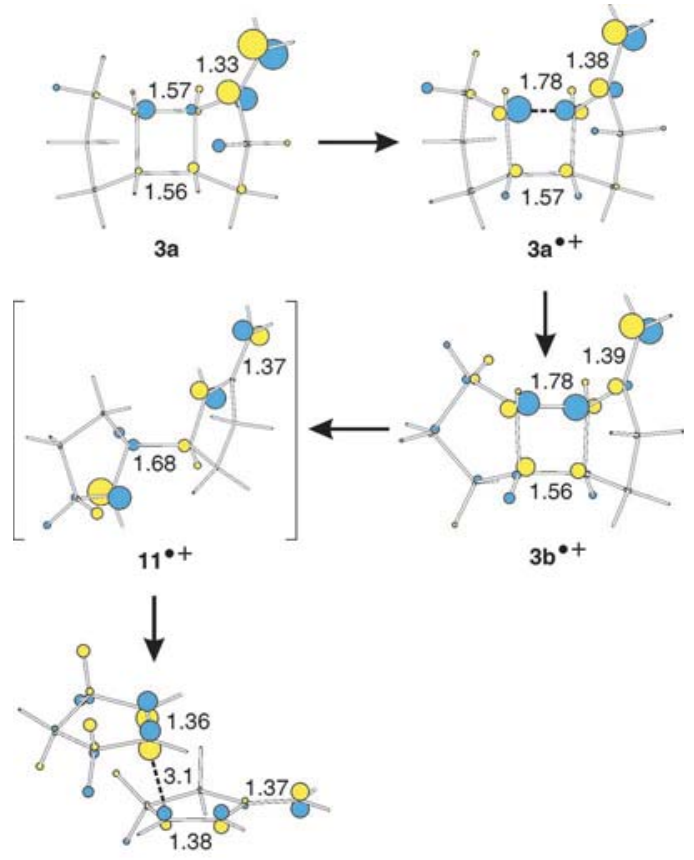

$12 \bullet+$

Figure 12. Species on the decay path of $\mathbf{3 a}$ upon ionization. Numbers indicate bond lengths in $\AA$. The MO shown is the HOMO, which is singly occupied in the radical cations.

tene and cyclopentene (see Figure 12). The overall process leading from the incipient radical cation $\mathbf{3} \mathbf{a}^{++}$to $\mathbf{1 2}^{\cdot+}$ is exergonic by $9.6 \mathrm{kcal} \mathrm{mol}^{-1}$, that is, it is thermochemically feasible (Figure 13).

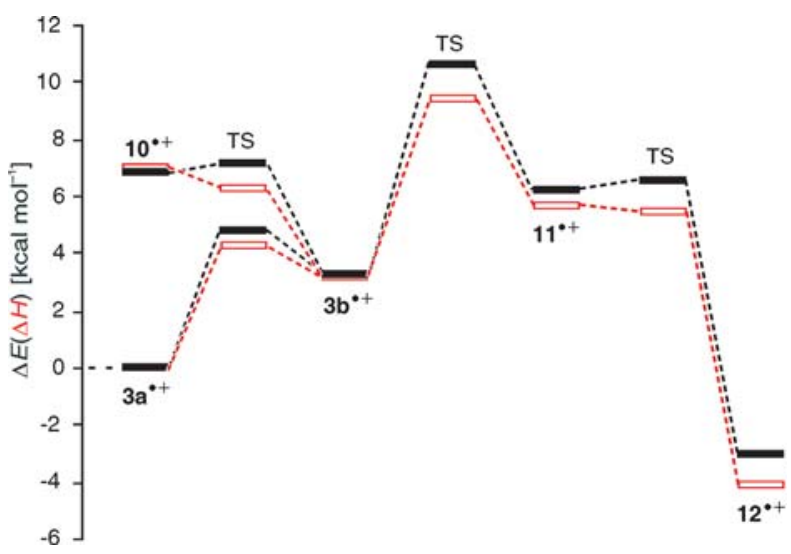

Figure 13. Energy (black) and enthalpy diagram (red) for the decay of $\mathbf{2 a}$ on ionization.

Complex $\mathbf{1 2}^{+}$is expected to show an intense low-energy charge transfer transition, and this is exactly what is predicted by TD-DFT calculations $\left(\lambda_{\max }=854 \mathrm{~nm}, f=0.188\right)$ and what is observed in the Ar matrix experiment. The IR spectra found after $\mathrm{X}$-irradiation of $\mathbf{3}$ in $\mathrm{Ar}$ are also in good accord with those predicted for $\mathbf{1 2}^{++}$(Figure 14) which corroborates the hypothesis that this species is formed in these 


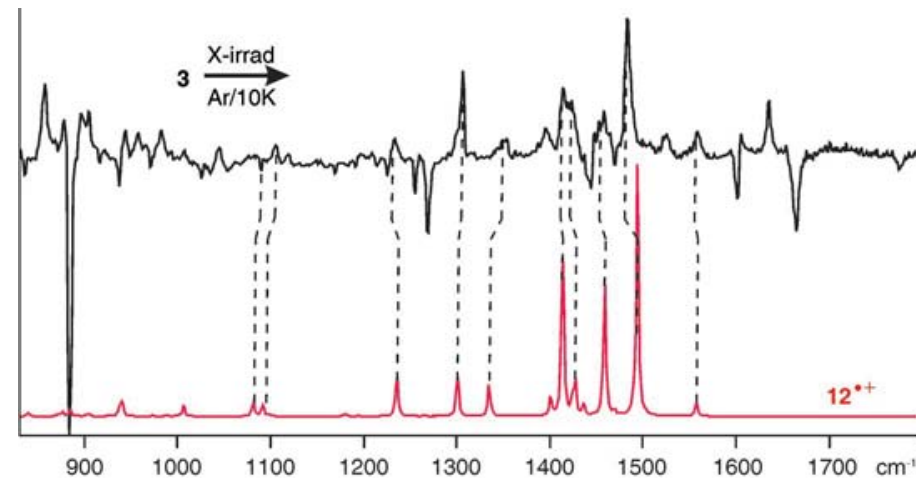

Figure 14. IR difference spectrum from Figure 3 (top) compared with that calculated by $\mathrm{B} 3 \mathrm{LYP} / 6-31 \mathrm{G}^{*}$ for the radical cation complex $\mathbf{1 2}^{+}$ (bottom).

experiments. Note that another radical cation with a maximum at about $620 \mathrm{~nm}$ is formed concomitantly and can be photoconverted to $\mathbf{1 2}^{-+}$. Most likely this is unrearranged $\mathbf{3} \mathbf{a}^{+}$and TD-DFT calculations predict indeed a transition at $668 \mathrm{~nm}(f=0.044)$ for this species.

Thus the picture with regard to the Ar matrix experiments is quite consistent: apparently, a part of the incipient parent radical cations $3 \mathbf{a}^{\cdot+}$ are endowed with enough excess energy to surmount the $9.4 \mathrm{kcal} \mathrm{mol}^{-1}$ barrier that is required for cleavage of the $\mathrm{C} 1-\mathrm{C} 2$ bond (which is followed promptly by dissociation of the $\mathrm{C} 6-\mathrm{C} 7$ bond) to yield the complex cation $\mathbf{1 2}^{+}$. The only question that remains is what happens on bleaching of $\mathbf{1 2}^{+}$(Figure 3, blue traces) because the product does not manifests itself palpably in the spectral regions we have investigated.

With regard to the PET experiments it is likely that the $9.4 \mathrm{kcal} \mathrm{mol}^{-1}$ barrier which is involved in the decay of $\mathbf{3} \mathbf{a}^{+}$ to $\mathbf{1 2}^{-+}$make that this process cannot compete with return electron transfer. The low quantum yield of PET-induced decomposition seems to indicate that this is indeed the case. However, even if some $\mathbf{1 2}^{\cdot+}$ was formed, this would have given 3-methylenecyclopentene and cyclopentenene on reneutralization, both compounds that would have escaped GC detection due to their high volatility.

\section{Conclusion}

Figure 15 sums up the findings of the present study, as well as of that on the fate of the tricyclic vinylcyclobutanes 1-3 in photoinduced electron transfer (PET) reactions. This fate depends critically on seemingly minor structural features such as the initial stereochemistry of the hydrocarbon and/ or the absence or presence of a methyl group. Oxidative cleavage of one of the four-membered ring bonds adjacent to the vinyl group (which leads to a species that profits from allylic resonance) can only occur if a tertiary carbonium ion can be formed in the process. Hence, in the presence of a methyl group on $\mathrm{C} 6$ it is invariably the $\mathrm{C} 2-\mathrm{C} 6$ bond that is cleft, in spite of the fact that the $\mathrm{C} 1-\mathrm{C} 2$ bond is lengthened

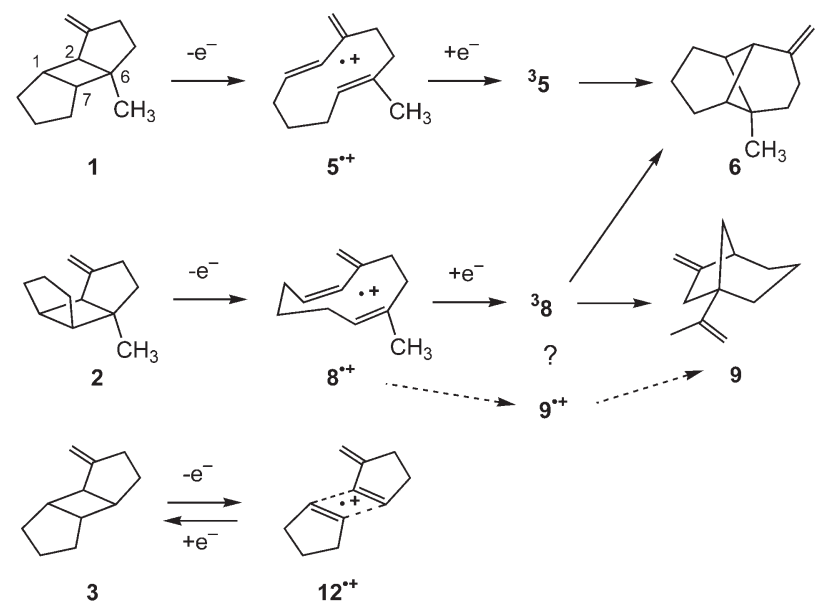

Figure 15. Summary of results of the present study and on the previous on ${ }^{[24,35]}$ on the photoinduced electron transfer (PET) chemistry of hydrocarbons 1-3.

in the incipient radical cations $\mathbf{1}^{\cdot+}$ and $\mathbf{2}^{\cdot+}$. This process is followed in both compounds by dissociation of the $\mathrm{C} 1-\mathrm{C} 7$ bond to yield a 1-methyl-8-methylene-1,6-cyclodecadiene radical cation $\left(5^{+} / \mathbf{8}^{++}\right)$the configuration of which with regard to the newly formed double bonds depends on the geometry of the reactant ( $E, E$ from $\mathbf{1}, Z, Z$ from $\mathbf{2}$ ).

Compounds $\mathbf{5}^{\cdot+}$ and $\mathbf{8}^{++}$are unambiguously identified as reactive intermediates in the Ar-matrix experiments. In the case of $\mathbf{1}$, the PET product is $\mathbf{6}$, a formal cross-cycloaddition product of $\mathbf{5}$. As formation of $\mathbf{6}^{\cdot+}$ from $\mathbf{5}^{+}$is thermochemically unfeasible, we assume that $\mathbf{6}$ arises after back electron transfer, probably from the triplet state of $\mathbf{5}$. In the case of $\mathbf{2}$ where one of the major PET products is hydrocarbon 9 we cannot decide from our experiments whether this is formed via $9^{+}$, followed by back electron transfer, or via ${ }^{3} \mathbf{8}$. Our search for a feasible pathway for the transformation $\mathbf{2}^{\cdot+} \rightarrow$ $\mathbf{9}^{+}+$did not turn up any rearrangements that could possibly compete with the rapid decay of $\mathbf{2}^{\cdot+}$ to $\mathbf{8}^{\cdot+}$.

In the absence of a methyl group on $\mathrm{C} 6$, cleavage of the C2-C6 bond is unfavorable and does not lead to stable products. Instead, $\mathbf{3}^{+}$undergoes stepwise cycloreversion to yield a complex between the radical cation of 1-methylene2-cyclopentadiene and cyclopentadiene $\left(\mathbf{1 2}^{+}+\right.$in Figure 15), which has been identified in an Ar matrix, mainly by the intense charge transfer band which is typical for such radical ion complexes. In PET experiments, $\mathbf{3}$ did not yield any detectable products, probably because the activation enthalpy for the cycloreversion to $\mathbf{1 2}^{+}\left(9.4 \mathrm{kcal} \mathrm{mol}^{-1}\right.$ according to B3LYP/6-31G* calculations) is too high for this process to compete successfully with back electron transfer which leads presumably back to 3 .

\section{Acknowledgements}

Support provided by the Deutsche Forschungsgemeinschaft and the Fonds der Chemischen Industrie is gratefully acknowledged. T.B. and 
K.P. are grateful for support by the Swiss National Science Foundation (project No 200020-105217) and J.G. thanks the Studienstiftung des Deutschen Volkes for a scholarship. The latter two sources of support made it possible for J.G. to visit Fribourg where she carried out most of the work described in this paper.

[1] M. Schmittel, A. Burghart, Angew. Chem. 1997, 109, 2658; Angew. Chem. Int. Ed. Engl. 1997, 36, 2551.

[2] M. Schmittel, M. K. Ghorai, in Electron Transfer in Chemistry, Vol. 2 (Ed.: V. Balzani), Wiley-VCH, Weinheim, 2001, p. 5.

[3] N. L. Bauld, Adv. Electron Transfer Chem. 1992, 2, 1.

[4] N. L. Bauld, D. J. Bellville, B. Harirchian, K. T. Lorenz, R. A. Pabon, D. W. Reynolds, D. D. Wirth, H. S. Chiou, B. K. Marsh, Acc. Chem. Res. 1987, 20, 371.

[5] J. P. Dinnocenzo, D. Conlon, J. Am. Chem. Soc. 1988, 110, 2324.

[6] J. P. Dinnocenzo, D. Conlon, Tetrahedron Lett. 1995, 36, 7415.

[7] T. Herbertz, H. D. Roth, J. Am. Chem. Soc. 1998, 120, 11904.

[8] P. G. Gassmann, Chemtracts: Org. Chem. 1989, 2, 24.

[9] P. A. Leber, J. E. Baldwin, Acc. Chem. Res. 2002, 35, 279.

[10] N. L. Bauld, J. Comput. Chem. 1990, 7, 896.

[11] R. A. Pabon, D. J. Belville, N. L. Bauld, J. Am. Chem. Soc. 1984, $106,2730$.

[12] J. Mattay, G. Trampe, J. Runsink, Chem. Ber. 1988, 121, 1991.

[13] J. Mattay, Angew. Chem. 1987, 99, 849; Angew. Chem. Int. Ed. Engl. 1987, 26, 825

[14] D. R. Arnold, X. Du, J. Am. Chem. Soc. 1989, 111, 7666.

[15] D. R. Arnold, X. Du, Can. J. Chem. 1994, 72, 403.

[16] H. D. Roth, H. Weng, D. Zhou, P. S. Lakkaraju, Acta Chim. Scand. 1997, 51, 626 .

[17] D. Zhou, M. Sheik, H. D. Roth, Tetrahedron Lett. 1996, 37, 2385.

[18] E. Haselbach, T. Bally, Z. Lanyiova, P. Baertschi, Helv. Chim. Acta 1979, 62, 583 .

[19] P. Jungwirth, T. Bally, J. Am. Chem. Soc. 1993, 115, 5783.

[20] Z. Zhu, T. Bally, J. Wirz, M. Fülscher, J. Chem. Soc. Perkin Trans. 2 1998, 1083.

[21] K. Schroeter, D. Schröder, H. Schwarz, G. Devi Reddy, O. Wiest, C. Carra, T. Bally, Chem. Eur. J. 2000, 6, 4422.

[22] T. Bally, S. Bernhard, S. Matzinger, J.-L. Roulin, G. N. Sastry, L. Truttmann, Z. Zhu, A. Marcinek, J. Adamus, R. Kaminski, J. Gebicki, F. Williams, G.-F. Chen, M. P. Fülscher, Chem. Eur. J. 2000, 6, 858.

[23] T. Bally, S. Bernhard, S. Matzinger, L. Truttmann, Z. Zhu, J.-L. Roulin, A. Marcinek, J. Gebicki, F. Williams, G.-F. Chen, H. D. Roth, T. Herbertz, Chem. Eur. J. 2000, 6, 849.

[24] J. Grota, J. Mattay, Photochem. Photobiol. Sci. 2005, 4, 625.
[25] J. Grota, I. Domke, I. Stoll, T. Schröder, J. Mattay, M. Schidtmann, H. Bögge, A. Müller, Synthesis 2005, 2321.

[26] A. D. Becke, J. Chem. Phys. 1992, 97, 9173.

[27] C. Lee, W. Yang, R. G. Parr, Phys. Rev. B 1988, 37, 785.

[28] Gaussian98, Revision A7-A11, M. J. Frisch, G. W. Trucks, H. B. Schlegel, G. E. Scuseria, M. A. Robb, J. R. Cheeseman, V. G. Zakrzewski, J. A. Montgomery, R. E. Stratmann, J. C. Burant, S. Dapprich, J. M. Millam, A. D. Daniels, K. N. Kudin, M. C. Strain, O. Farkas, J. Tomasi, V. Barone, M. Cossi, R. Cammi, B. Mennucci, C. Pommelli, C. Adamo, S. Clifford, J. Ochterski, G. A. Petersson, P. Y. Ayala, Q. Cui, K. Morokuma, D. K. Malick, A. D. Rabuck, K. Raghavachari, J. B. Foresman, J. Cioslowski, J. V. Ortiz, B. B. Stefanov, G. Liu, A. Liashenko, P. Piskorz, I. Komaromi, R. Gomperts, R. L. Martin, D. J. Fox, T. Keith, M. A. Al-Laham, C. Y. Peng, A. Nanayakkara, M. Challacombe, P. M. W. Gill, B. G. Johnson, W. Chen, M. W. Wong, J. L. Andres, C. Gonzales, M. Head-Gordon, E. S. Repogle, J. A. Pople, Gaussian, Inc., Pittsburgh, PA, 1998.

[29] M. E. Casida, in Recent Advances in Density Functional Methods, Part I (Ed.: D. P. Chong), World Scientific, Singapore, 1995, p. 155.

[30] R. E. Stratmann, G. E. Scuseria, M. J. Frisch, J. Chem. Phys. 1998 $109,8218$.

[31] The signal for the proton at $\mathrm{C} 7(\delta=2.103$, dd, $1 \mathrm{H}, J=7.5,7.5 \mathrm{~Hz})$ shows two couplings to its three vicinal protons, while the signal for the proton at $\mathrm{C} 1(\delta=2.214$, ddd, $1 \mathrm{H}, J=7.3,7.2,3.8 \mathrm{~Hz})$ shows three couplings to its four vicinal protons. Both patterns require that the dihedral angle between two of the three or four $\mathrm{C}-\mathrm{H}$ bonds, respectively, must be close to $90^{\circ}$. This situation occurs in conformations $\mathbf{1 a}(1-\mathrm{H}$ to one of the two $10-\mathrm{H}$ protons) and $\mathbf{1 b}(7-\mathrm{H}$ to one of the 8-H protons), but not in $\mathbf{1 c}$ and $\mathbf{1 d}$, where a broadening of the ${ }^{1} \mathrm{H}$ NMR signals would be expected. Hence we can assume that at room temperature mainly conformers $\mathbf{1 a}$ and $\mathbf{1 b}$ are present.

[32] P. Jungwirth, P. Carsky, T. Bally, J. Am. Chem. Soc. 1993, 115, 5776.

[33] Note that the very low frequency libration modes in this complex may lead to an overestimation of the entropy increase. However, the process is also exothermic by $4.1 \mathrm{kcal} \mathrm{mol}^{-1}$ and therefore it is certainly thermodynamically feasible.

[34] N. Bülow, W. A. König, Phytochemistry 2000, 55, 141.

[35] J. Grota, PhD thesis, University of Bielefeld (Germany), 2004.

[36] In $6^{+}$one of the four-membered ring bonds that is adjacent to the exocyclic methylene group is also significantly elongated (to $1.79 \AA$ ). However, $\mathbf{6}^{+}$exists only as a single conformer.

Received: October 27, 2005 Revised: February 14, 2006 Published online: April 5, 2006 